\title{
Reversible feedback confinement
}

\author{
Léo Granger ${ }^{1} \quad$ Luis Dinis $^{1} \quad$ Jordan M. Horowitz ${ }^{2} \quad$ Juan MR Parrondo $^{1}$ \\ September 22, 2016 \\ ${ }^{1}$ Departamento de Física Atómica, Molecular y Nuclear and GISC, Universidad Complutense Madrid, \\ 28040 Madrid, Spain \\ ${ }^{2}$ Department of Physics, Physics of Living Systems, Massachusetts Institute of Technology, Cambridge, \\ MA 02139, USA
}

\begin{abstract}
We present a feedback protocol that is able to confine a system to a single micro-state without heat dissipation. The protocol adjusts the Hamiltonian of the system in such a way that the Bayesian posterior distribution after measurement is in equilibrium. As a result, the whole process satisfies feedback reversibility - the process is indistinguishable from its time reversal - and assures the lowest possible dissipation for confinement. In spite of the whole process being reversible it can surprisingly be implemented in finite time. We illustrate the idea with a Brownian particle in a harmonic trap with increasing stiffness and present a general theory of reversible feedback confinement for systems with discrete states.
\end{abstract}

PACS 05.70.Ln - Nonequilibrium and irreversible thermodynamics

PACS 05.40.-a - Fluctuation phenomena, random processes, noise, and Brownian motion

PACS 05.70.-a - Entropy thermodynamics

Micro-manipulation techniques introduced in the last decades - optical and magnetic tweezers, monitored quantum dots, or atomic force microscopy - call for a theoretical framework to analyze driven systems at the micro-scale. An important element in such a framework is feedback control, where the system is monitored or measured at certain stages of a process, and the driving protocol depends on the outcomes of the measurements 11. Feedback control can be used, for instance, to increase the performance of certain devices, such as Brownian ratchets [2,3] and micro-motors [4], or to diminish thermal fluctuations, confining the system to a small region of phase space. A particular case of the latter is feedback cooling, a technique that has been implemented in classical and quantum systems $[5 \sqrt{8}$ and plays a relevant role in quantum optomechanics 9 .

Now, it is well established that for any feedback protocol the information itself is a thermodynamic resource, which is quantified within the framework of information thermodynamics [10]. In particular, the work $W$ needed to perform an isothermal feedback process at temperature $T$ is bounded by $10-21$

$$
W \geq \Delta F-k T I,
$$

where $k$ is Boltzmann's constant, $\Delta F$ is the difference in free energy between the final and initial states of the system, and $I$ is the amount of information gained by the measurement. For a single feedback loop, $I$ is the information-theoretic mutual information between the measurement outcome and the system's microstate 10,11 . As a consequence of (1), the work $W$ performed in a feedback process might be lower than the variation in the free energy of the system $\Delta F$.

Processes saturating bound (1) are of particular relevance as they make an optimal use of information. For a single measurement, such processes were identified and characterized in 22 24, where it is shown that optimal processes must be reversible under feedback. For systems in contact with a thermal reservoir, reversibility requires that the probabilistic state of the system conditioned on the measurement outcome be the equilibrium Gibbs state at all times. In particular, after a measurement, the probabilistic state changes abruptly: it is updated via Bayesian inference using the information provided by the measurement [10. To achieve reversibility, the Hamiltonian of the system must be tuned so that its Gibbs distribution coincides with the measurement-dependent posterior probability distribution [25.26].

In this letter, we apply this recipe to design an optimal, reversible feedback protocol that confines a system in contact with a thermal bath to a small region of phase space or even to a single micro-state. In any such confinement procedure, the free energy change is $\Delta F=\Delta E-T \Delta S$ where $\Delta E$ is the change 
of internal energy of the system and $\Delta S$ the change in the entropy, which is negative. Hence, (1) can be rewritten as

$$
Q_{\text {diss }}=W-\Delta E \geq-T \Delta S-k T I .
$$

Without feedback $(I=0)$, confinement must be accompanied by dissipated heat $Q_{\text {diss }} \geq-T \Delta S>0$, which is a direct consequence of the second law 25,27]. This dissipation, however, can be decreased if we make use of feedback. Here we introduce an optimal feedback confining protocol consisting of a series of measurements, each followed by a rapid tuning of the Hamiltonian that leaves the system in equilibrium. Between two consecutive measurements the Hamiltonian is kept constant. Reversibility ensures that equality is met in (22. In addition, since the probabilistic state of the system does not change between measurements, the amount of heat dissipated to the environment is zero. Hence, we are able to design a general protocol where the reduction in the system's entropy is entirely due to the acquisition of information, $-\Delta S=k I$.

We first illustrate this idea with a simple example, similar to the one introduced in [4]. Consider an overdamped Brownian particle with position $z(t)$ in a harmonic potential $V_{\kappa, y}(z)=\frac{1}{2} \kappa(z-y)^{2}$ with tunable center $y$ and stiffness $\kappa$. The particle's dynamics obey the Langevin equation $\gamma \dot{z}=-\kappa(z-y)+\xi_{t}$, where $\gamma$ is the friction coefficient and $\xi_{t}$ is zero-mean Gaussian white noise with covariance $\left\langle\xi_{t} \xi_{t^{\prime}}\right\rangle=2 \gamma k T \delta\left(t-t^{\prime}\right)$ verifying the fluctuation-dissipation theorem.

In the absence of control, the particle's position relaxes to equilibrium with Gibbs distribution,

$$
\rho_{\kappa, y}(z) \propto \exp \left(-\frac{V_{\kappa, y}(z)}{k T}\right)
$$

which, in this case, is Gaussian with mean $\bar{z}=y$ and variance $\sigma^{2}=k T / \kappa$. Our goal is then to confine the particle to a smaller region of space by manipulating the control parameters $\lambda=(\kappa, y)$ using feedback.

To implement the feedback, we preform an imperfect measurement of the particle's position, yielding outcome $m$ with distribution $q(m \mid z)$ depending on the particle's true position $z$. Here, we make the standard assumption [4] of Gaussian errors, where $m$ is drawn from the Gaussian distribution about $z$,

$$
q(m \mid z) \propto \exp \left(-\frac{(m-z)^{2}}{2 \sigma_{\mathrm{m}}^{2}}\right),
$$

where the standard deviation $\sigma_{\mathrm{m}}$ quantifies the error in the measurement.

The particle's post-measurement distribution $\rho^{\prime}$ is obtained from the pre-measurement distribution $\rho$ via Bayes' theorem:

$$
\rho^{\prime}(z \mid m)=\frac{q(m \mid z) \rho(z)}{\pi(m)},
$$

where $\pi(m)=\int q(m \mid z) \rho(z) \mathrm{d} z$ is the marginal distribution of measurement outcomes. For the control to be reversible, we need to adjust our control parameters $\lambda$ immediately after the measurement, so that $\rho^{\prime}$ is the new equilibrium state. It is straightforward to check that, after a measurement characterized by the Gaussian distribution (4), a Gaussian pre-measurement state, like (3), centered at $\bar{z}$ with variance $\sigma^{2}$, updates to a post-measurement state according to 5 that is also Gaussian with mean and variance [4]

$$
\begin{aligned}
\bar{z}^{\prime}(m) & =\frac{\sigma_{\mathrm{m}}^{2}}{\sigma^{2}+\sigma_{\mathrm{m}}^{2}} \bar{z}+\frac{\sigma^{2}}{\sigma^{2}+\sigma_{\mathrm{m}}^{2}} m \\
\frac{1}{\sigma^{\prime 2}} & =\frac{1}{\sigma^{2}}+\frac{1}{\sigma_{\mathrm{m}}^{2}}
\end{aligned}
$$

Thus, we can make the post-measurement distribution an equilibrium distribution by setting the stiffness and the center of the trap to the new values $\kappa^{\prime}=k T / \sigma^{2}$ and $y^{\prime}(m)=\bar{z}^{\prime}(m)$.

The effect of repeating this reversible feedback procedure at successive times $t_{1}, \ldots, t_{n}$ is to confine the particle at a well-defined position. In fact, (7) implies that the post-measurement stiffness $\kappa^{\prime}=\kappa+k T / \sigma_{\mathrm{m}}^{2}>\kappa$ 

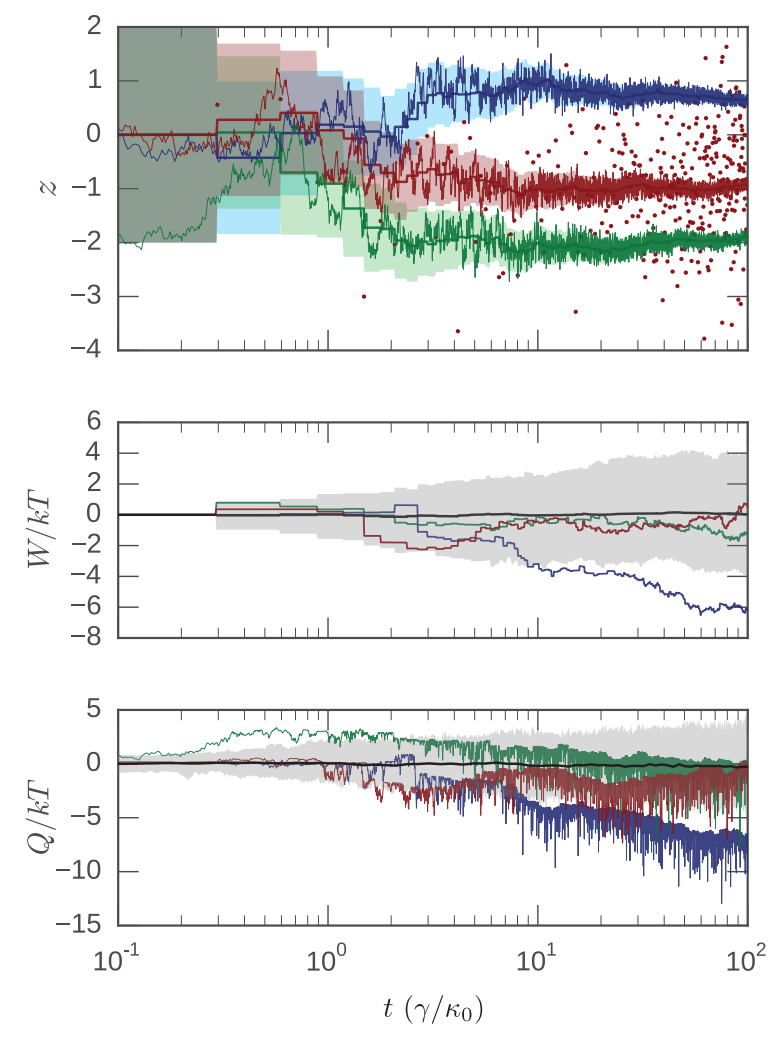

Figure 1: (Color online) Top panel: Three realizations (blue, red, green) of reversible confinement for a harmonically-trapped Brownian particle. The strongly-fluctuating thin lines represent the particle's timedependent position $z(t)$; the thick lines are the trap centers $y_{t}$; and the shaded area corresponds to $y_{t} \pm 2 \sigma_{t}$, where $\sigma_{t}=k T / \kappa_{t}$ is the equilibrium variance. The scatter of red dots are the red trajectory's measurement outcomes. Measurements are performed every $\Delta t=0.3\left(\gamma / \kappa_{0}\right)$ with error $\sigma_{\mathrm{m}}^{2}=\sigma_{0}^{2}=1$. Middle panel: Work performed during the confinement process. The black line is the average over 100 realizations and the gray shaded area is the middle $90 \%$ of the work values. The colored lines correspond to the realizations depicted in the top panel. Bottom panel: Same as middle for the heat released into the environment. 
increases by $k T / \sigma_{\mathrm{m}}^{2}>0$ in each reversible feedback step. Therefore, the post-measurement stiffness $\kappa_{n}$ after the $n^{\text {th }}$ measurement at time $t_{n}$ is

$$
\kappa_{n}=\kappa_{0}+n \frac{k T}{\sigma_{\mathrm{m}}^{2}}
$$

where $\kappa_{0}$ is the initial stiffness of the trap. Consequently, the post-measurement variance of the particle $\sigma_{n}^{2}=k T / \kappa_{n}$ decreases to zero as the number of measurements increases.

All along the process the average potential energy of the particle is constant and equal to $\frac{1}{2} k T$, by the equipartition theorem, so $\Delta E=0$. In addition, as discussed above, since the potential is instantaneously modified to leave the particle at equilibrium, there is no subsequent evolution nor relaxation between measurements and $Q=0$. Hence, the first law implies $W=0$ on average.

Alternatively, we can confirm there is zero heat dissipation and that the process is reversible directly from the second law by computing the mutual information between $m$ and $z$ in one measurement,

$$
I(m, z)=\int d m d z \pi(m) q(m \mid z) \log \frac{q(m \mid z)}{\pi(m)},
$$

and demonstrating its equality with $-\Delta S$.

In our example, $q(m \mid z), \rho(z)$ and $\pi(m)$ are Gaussian distributions of variances $\sigma_{m}^{2}, \sigma^{2}$ and $\sigma_{m}^{2} \sigma^{2} / \sigma^{\prime 2}$. Using the entropy of a Gaussian distribution $\rho(X), h(\rho)=\log \left(\sigma_{x} \sqrt{2 \pi e}\right)$, it is straight forward to show that

$$
I(m, z)=\frac{1}{2} \log \frac{\sigma^{\prime 2}}{\sigma^{2}} .
$$

On the other hand, after a measurement, the state of the system updates from a Gaussian of variance $\sigma^{2}$ to a Gaussian of variance $\sigma^{\prime 2}$ as shown in (7). Hence, the entropy of the particle changes as

$$
\begin{aligned}
\Delta S & =-k\left(\log \left(\sigma^{\prime} \sqrt{2 \pi e}\right)-\log (\sigma \sqrt{2 \pi e})=-k \frac{1}{2} \log \frac{\sigma^{\prime 2}}{\sigma^{2}}\right. \\
& =-k I(m, z) .
\end{aligned}
$$

Since the particle is always at equilibrium, the second law (2) applies with an equal sign and $Q=0$.

The optimal protocol thus ensures that no work is performed nor heat is dissipated on average. On the other hand, both would diverge for any non-feedback protocol that squeezes the trap potential to a single point, since in this case $\Delta S$ is infinity. Another important feature is that the confinement can be realized as fast as we like: the potential is updated immediately after each measurement and the system's probabilistic state, being always in equilibrium, does not change between measurements. Hence, there is no need of running the protocol slowly to achieve reversibility.

Three realizations of the trapping process with imprecise measurements $\left(\sigma_{\mathrm{m}}^{2}=\sigma_{0}^{2}=1\right)$ are depicted on Fig. 1. The top panel shows the time evolution of the position of the particle (thin strongly-fluctuating lines) and of the trap (thick lines). For the realization ending in the middle (red), we also show the measurement outcomes. Even though the measurement is not very precise, confinement is achieved eventually. In fact, each measurement provides a piece of information, even if it might be small. The middle and the bottom panels respectively depict the work performed on the particle and the heat released to the environment. On these panels, the black line represents the average over 100 realizations and the gray shaded area encompasses the middle $90 \%$ of work and heat values (notice that the blue realization is atypical from an energetic point of view). We indeed verify that the average heat and work are zero during the process.

We now turn to the formulation of a general theory of reversible feedback confinement for a system with discrete phase space $\Gamma$ in contact with a heat bath at temperature $T$. The generalization to continuous phase space is straightforward. Assume that the energy $E(z \mid \lambda)$ of any micro-state $z \in \Gamma$ can be controlled through the parameter (or set of parameters) $\lambda$. As in our example, the system is initially in equilibrium, with control parameter set to an initial value $\lambda=\lambda_{0}$. Then, at times $t_{1}, \ldots, t_{N}$ we measure a system observable $M=M(z)$ with outcomes drawn from the set $m \in \mathcal{M}$ according to the distribution $q(m \mid z)$, followed by a measurement-dependent switch of $\lambda$ to perform a reversible feedback process. 
After the $n^{\text {th }}$ measurement, with outcome $m_{n}$, the post-measurement distribution $p_{n}$ obtained from the pre-measurement state $p_{n-1}$ according to Bayes' theorem is

$$
p_{n}\left(z \mid \mu_{n}\right)=\frac{q\left(m_{n} \mid z\right) p_{n-1}\left(z \mid \mu_{n-1}\right)}{\pi_{n}\left(m_{n} \mid \mu_{n-1}\right)},
$$

where $\mu_{n} \equiv\left(m_{1}, \ldots, m_{n-1}, m_{n}\right)$ is the list of the first $n$ measurement outcomes and $\pi_{n}\left(m_{n} \mid \mu_{n-1}\right)=$ $\sum_{z} q\left(m_{n} \mid z\right) p_{n-1}\left(z \mid \mu_{n-1}\right)$ is the probability that the outcome of the $n^{\text {th }}$ measurement is $m_{n}$ given the list $\mu_{n-1}$ of the first $n-1$ outcomes. Notice that here we explicitly indicate the dependence of the posterior distribution $p_{n}\left(z \mid \mu_{n}\right)$ on the whole history of measurements $\mu_{n}$ [cf. (5)].

The reversible feedback condition dictates that immediately after the measurement the control parameter is changed to a value $\lambda\left(\mu_{n}\right)$ such that the post-measurement distribution 12 is equilibrium:

$$
E\left(z \mid \lambda\left(\mu_{n}\right)\right)=-k T \log p_{n}\left(z \mid \mu_{n}\right)+F_{n}\left(\mu_{n}\right),
$$

where $F_{n}\left(\mu_{n}\right)$ is the free energy of the system after the switch. Its value is arbitrary and can be changed by redefining the reference energy, affecting only the work and not the dissipated heat. Between two measurements, the control parameter is held constant. Since the system is in equilibrium, the distribution $p_{n}$ is invariant between times $t_{n}^{+}$and $t_{n+1}^{-}$. Therefore it is also the pre-measurement distribution of the subsequent $(n+1)^{\text {th }}$ measurement.

The effect of the procedure described above is to eventually confine the system into one single microstate (or set of micro-states) without dissipating heat to the environment. This confinement relies on the following remarkable property of the process, which we will demonstrate in the following (cf. (14) to (16)): given the final micro-state $z_{N}=z\left(t_{N}\right)$, the measurement outcomes in the sequence $\mu_{N}=\left(m_{1}, \ldots, m_{N}\right)$ are independent and identically dnistributed (i.i.d.) according to $q\left(m \mid z_{N}\right)$. Thus, by the law of large numbers, when the number of measurements $N$ is large, the empirical frequency $\nu_{m}$ of outcome $m$ - that is the fraction of times $m$ appears in the list $\mu_{N}$ - is close to $q\left(m \mid z_{N}\right)$. As a consequence, just knowing the empirical distribution $\nu=\left\{\nu_{m}\right\}$ of the list $\mu_{N}$ of measurement outcomes, we can infer with certainty that the final micro-state $z_{N}$ is in the set of micro-states that verify $q\left(m \mid z_{N}\right) \simeq \nu_{m}$ for all $m$. If the distributions $q(m \mid z)$ are different for all micro-states $z$, then the system is confined to a single micro-state. On the other hand, if there exist two micro-states $z^{\prime}$ and $z^{\prime \prime}$ such that $q\left(m \mid z^{\prime}\right)=q\left(m \mid z^{\prime \prime}\right)$, then the two states are indistinguishable to the observer, and our protocol confines the system to the set $\left\{z^{\prime}, z^{\prime \prime}\right\}$. To arrive at these conclusions, we now calculate the distribution $p_{N}(z \mid \nu)$ of $z_{N}$ conditioned on the empirical density $\nu$ and show that it is highly peaked.

To begin, we apply 12 recursively:

$$
p_{N}\left(z \mid \mu_{N}\right)=\frac{p_{0}(z) \prod_{n=1}^{N} q\left(m_{n} \mid z\right)}{\pi_{N}\left(m_{N} \mid \mu_{N-1}\right) \ldots \pi_{1}\left(m_{1}\right)},
$$

where $p_{0}(z)$ is the initial equilibrium distribution. The denominator (14) is simply the probability to observe the sequence $\mu_{N}$. Therefore, the joint probability of observing $\mu_{N}$ and ending the process at $z_{N}=z$ reads

$$
\mathcal{P}_{N}\left(z, \mu_{N}\right)=p_{0}(z) \prod_{n=1}^{N} q\left(m_{n} \mid z\right)
$$

Summing over all possible $\mu_{N}$, we obtain the marginal distribution of $z_{N}, p_{N}(z)=p_{0}(z)$, which remarkably is constant along the whole process. Thus, the probability of $\mu_{N}$, given the final micro-state $z_{N}$ is

$$
P_{N}\left(\mu_{N} \mid z_{N}\right)=\prod_{n=1}^{N} q\left(m_{n} \mid z_{N}\right)=\prod_{m \in \mathcal{M}} q\left(m \mid z_{N}\right)^{N \nu_{m}} .
$$

That is, given the final state $z_{N}$, the measurement outcomes are i.i.d., so that the probability to observe a specific sequence $\mu_{N}$ depends only on the empirical distribution $\nu=\left\{\nu_{m}\right\}$. The probability to observe an 
empirical distribution $\nu$, given $z_{N}$, is obtained by multiplying $(16)$ by the number of sequences yielding the same distribution,

$$
P_{N}\left(\nu \mid z_{N}\right)=\frac{N !}{\prod_{m}\left(N \nu_{m}\right) !} P_{N}\left(\mu_{N} \mid z_{N}\right) \approx e^{-N \epsilon\left(\nu, z_{N}\right)}
$$

for $N$ large, where we used Stirling's approximation and defined $\epsilon(\nu, z)=D\left[\nu_{m} \| q(m \mid z)\right]=\sum_{m} \nu_{m} \log \frac{\nu_{m}}{q(m \mid z)}$, the relative entropy between the empirical distribution $\nu$ and $q(m \mid z)$ 28]. As a relative entropy, $\epsilon=0$ only when $\nu_{m}=q(m \mid z)$. Notice that $\epsilon\left(\nu, z_{N}\right)$ is the large deviation rate function for the empirical distribution $\nu$ [29].

Finally, applying again Bayes' theorem, we get the probability that the final state is $z_{N}=z$ given the empirical distribution $\nu$ of measurement outcomes,

$$
p_{N}(z \mid \nu)=\frac{P_{N}(\nu \mid z) p_{0}(z)}{\sum_{z^{\prime}} P_{N}\left(\nu \mid z^{\prime}\right) p_{0}\left(z^{\prime}\right)} \approx \frac{p_{0}(z) e^{-N \epsilon(\nu, z)}}{\sum_{z^{\prime}} p_{0}\left(z^{\prime}\right) e^{-N \epsilon\left(\nu, z^{\prime}\right)}} .
$$

For this distribution to be equilibrium, the final energy landscape must be

$$
E\left(z \mid \lambda\left(\mu_{N}\right)\right)=E\left(z \mid \lambda_{0}\right)+N k T \epsilon(\nu, z) .
$$

For large $N$, the system becomes trapped in the most likely micro-state(s) $z^{*}$ that minimize $\epsilon\left(\nu, z^{*}\right)$. Those are exactly the states for which the empirical frequencies $\nu_{m}$ approach $q\left(m \mid z^{*}\right)$, making $\epsilon\left(\nu, z^{*}\right) \rightarrow 0$. As in our example, the probabilistic state of the system is constant between measurements and therefore the average dissipated heat is zero.

We have shown that it is possible to use feedback to confine a system with zero average heat, even when the measurements have large errors. In contrast, to achieve the same confinement without feedback, one has to dissipate an enormous amount of heat.

One of the main novelties of the present work is the consideration of errors in the measurement. With error-free measurements, confinement without dissipation is trivial. However, we have shown that confinement is still possible no matter how inaccurate the measurements are; although for less precise measurements more are needed to achieve perfect confinement. For instance, in our Brownian particle example, the variance of the particle after $n$ measurements can be obtained by recurring (7) giving $\left(\sigma^{n}\right)^{2}=\sigma_{m}^{2} \sigma_{0}^{2} /\left(n \sigma_{0}^{2}+\sigma_{m}^{2}\right)$, with $\sigma_{0}^{2}$ the initial variance of the particle. For large $n,\left(\sigma^{n}\right)^{2} \approx \sigma_{m}^{2} / n$. Thus, to confine the particle, that is, to reduce particle variance below a small target value $\left(\sigma^{n}\right)^{2}<\delta^{2}$, the number of measurements needed are proportional to the measurement error $\sigma_{m}^{2}: n \gtrsim \sigma_{m}^{2} / \delta^{2}$.

Errors in the measurements can also give rise to counter-intuitive results, such as providing better discrimination of the system's micro-states. Consider, for instance, a particle in a one-dimensional box of length 1 and a binary measurement with $m \in\{\mathrm{L}, \mathrm{R}\}$, standing for the left and right half of the box, respectively. Our protocol with an error-free measurement, $q(\mathrm{R} \mid z)=1$ if $z>1 / 2$ and zero otherwise and $q(\mathrm{~L} \mid z)=1-q(\mathrm{R} \mid z)$, will confine the particle to one of the two halves of the container and will not be able to discriminate micro-states within each half of the container. Notice that in this case, although measurement outcome $\{L, R\}$ depends on the state $z$, there are a number of different states that share a common outcome probability distribution $q(m \mid z)$ and, as stated above, the observer cannot distinguish among them. On the other hand, a binary measurement with $q(\mathrm{R} \mid z)=z$ and $q(\mathrm{~L} \mid z)=1-z$ is able to confine the particle to a single point with zero heat.

We conclude with some remarks. First, while the system dissipates no heat, there is an energetic cost to acquiring or, alternatively, to erasing the information [10, 11, 30 32]. There is no avoiding the second law, we simply have shifted its cost to the memory device. However, such a shift is relevant when we need to keep low the heat dissipated to the surroundings of the controlled system 33, 34.

Second, although the final state of any single realization is tightly confined, that final state is a priori random: the marginal distribution of the system at the end of the confinement process is the same as the initial distribution. The trade-off is that our protocol can be implemented at arbitrary speed without compromising reversibility. The reason is that the statistical state of the system is frozen between measurements, so that the system never actually relaxes towards equilibrium. Hence, its relaxation time is irrelevant and 
the speed of the process is simply given by the frequency of the measurements. However, if we wished to confine the system to a fixed pre-determined low-entropy state, as required for feedback cooling [1, 27, our protocol requires one last slow isothermal step that drags the system to the target state. To be precise, even though the relaxation time to equilibrium can be avoided using our confinement protocol, the speed of the process could be still limited by other factors, for instance, the time needed to modify the Hamiltonian experimentally. In many systems though, like a colloidal particle trapped in a laser beam for example, the tuning of the Hamiltonian can actually be done much faster than the position relaxation time of the particle. Our protocol would then ensure a very low dissipation at fast operation times.

Lastly, our protocol requires fine-tuned control over the Hamiltonian to fulfill $(13)$. This can be a hinderance if we aim to decrease the fluctuations of the velocity of a classical particle [35, 36, since it is difficult to construct velocity-dependent forces in the lab. This difficulty could be overcome in the quantum version of our procedure, since fine-tuned control over the energy-levels of small quantum systems is often more feasible. For instance, increasing the stiffness of a harmonic trap in the quantum regime, affects both the kinetic and the potential energy of the oscillator. Regarding the fine-tuning of the Hamiltonian needed for confinement, our example provides also an interesting lesson. As indicated by equation (8), there is a trade-off between the measuring error and the fractional change of the control parameter needed to adjust the Hamiltonian at each step. Therefore, when it is experimentally difficult to slightly modify the control parameter, our result suggest this could be overcome also by increasing measuring precision.

Interestingly, our protocol being reversible can also be employed to extract the maximum amount of work from the information provided by a number of measurements, it suffices to perform an isothermal quasistatic (reversible) transformation from the confined state back to the initial unconfined state, i.e., a sort of expansion. During reversible confinement without work, the system increases its free energy in an amount $\Delta F=k T I$. By expanding reversibly and isothermally (without any further measurement) all this free-energy is converted into (extracted) work $W=-\Delta F=-k T I$. In the first part of the operation, the optimal feedback transforms the information contained in fluctuations into free energy but, by confining the system, reduces the fluctuations exhausting the amount of information. Then, during work extraction, heat is allowed to enter the system which in turn develops new fluctuations that can be exploited in the next cycle of operation [4, 37, 40].

This work has been supported by grants ENFASIS (FIS2011-22644) and TerMic (FIS2014-52486-R) from the Spanish Government. JMH is supported by the Gordon and Betty Moore Foundation through Grant GBMF4343.

\section{References}

[1] H. Touchette and S. Lloyd. Information-theoreitc limits of control. Phys. Rev. Lett., 84(6):1156-1159, 2000.

[2] Francisco J. Cao, Luis Dinis, and Juan M. R. Parrondo. Feedback Control in a Collective Flashing Ratchet. Phys. Rev. Lett., 93(4):040603, July 2004.

[3] Benjamin Lopez, Nathan Kuwada, Erin Craig, Brian Long, and Heiner Linke. Realization of a Feedback Controlled Flashing Ratchet. Phys. Rev. Lett., 101(22), November 2008.

[4] D. Abreu and U. Seifert. Extracting work from a single heat bath through feedback. EPL, 94(1):10001, April 2011.

[5] Tongcang Li, Simon Kheifets, and Mark G Raizen. Millikelvin cooling of an optically trappedmicrosphere in vacuum. Nature Physics, 7(7):527-530, March 2011.

[6] Jan Gieseler, Bradley Deutsch, Romain Quidant, and Lukas Novotny. Subkelvin Parametric Feedback Cooling of a Laser-Trapped Nanoparticle. Phys. Rev. Lett., 109(10):103603, September 2012.

[7] A.E. Cohen. Control of nanoparticles with arbitrary two-dimensional force fields. Phys. Rev. Lett., 94:118102, 2005. 
[8] A.E. Cohen and W.E. Moerner. Suppressing brownian motion of individual biomolecules in solution. Proc. Natl. Acad. Sci., 103(12):4362-4365, 2006.

[9] Markus Aspelmeyer, Tobias J Kippenberg, and Florian Marquardt. Cavity optomechanics. Rev Mod Phys, 86(4):1391-1452, December 2014.

[10] Juan M R Parrondo, Jordan M Horowitz, and Takahiro Sagawa. Thermodynamics of information. Nature Physics, 11(2):131-139, February 2015.

[11] Takahiro Sagawa and Masahito Ueda. Second Law of Thermodynamics with Discrete Quantum Feedback Control. Phys. Rev. Lett., 100(8):080403, February 2008.

[12] F. J. Cao and M. Feito. Thermodynamics of feedback controlled systems. Phys. Rev. E, 79(4):041118, April 2009.

[13] Armen E. Allahverdyan, Dominik Janzing, and Guenter Mahler. Thermodynamic efficiency of information and heat flow. J. Stat. Mech., 2009(09):P09011, September 2009.

[14] Jordan M. Horowitz and Suriyanarayanan Vaikuntanathan. Nonequilibrium detailed fluctuation theorem for repeated discrete feedback. Phys. Rev. E, 82(6):061120, December 2010.

[15] Youhei Fujitani and Hiroyuki Suzuki. Jarzynski Equality Modified in the Linear Feedback System. Journal of the Physical Society of Japan, 79(10):104003, October 2010.

[16] M. Ponmurugan. Generalized detailed fluctuation theorem under nonequilibrium feedback control. Physical Review E, 82(3), September 2010.

[17] Sosuke Ito and Takahiro Sagawa. Information Thermodynamics on Causal Networks. Phys. Rev. Lett., 111(18):180603, October 2013.

[18] Naoto Shiraishi and Takahiro Sagawa. Fluctuation theorem for partially masked nonequilibrium dynamics. Physical Review E, 91(1):012130, January 2015.

[19] J. M. Horowitz and M. Esposito. Thermodynamics with continuous information flow. Phys. Rev. X, 4:031015, 2014.

[20] D. Hartich, A. C. Barato, and U. Seifert. Stochastic thermodynamics of bipartite systems: transfer entropy inequalities and a Maxwell's demon interpretation. J. Stat. Mech., 2014(2):P02016, February 2014.

[21] Léo Granger. Irreversibility and information. PhD thesis, Technische Universität Dresden, Dresden, Germany, November 2013.

[22] Jordan M. Horowitz and Juan M. R. Parrondo. Thermodynamic reversibility in feedback processes. EPL, 95(1):10005, July 2011.

[23] Jordan M. Horowitz and Juan M. R. Parrondo. Designing optimal discrete-feedback thermodynamic engines. New J. Phys., 13(12):123019, December 2011.

[24] Jordan M. Horowitz and Juan M. R. Parrondo. Optimizing Non-ergodic Feedback Engines. Acta Physica Polonica B, 44:803, May 2013.

[25] M. Esposito and C. Van den Broeck. Second law and Landauer principle far from equilibrium. EPL, 95(4):40004, August 2011.

[26] Kurt Jacobs. Quantum measurement and the first law of thermodynamics: The energy cost of measurement is the work value of the acquired information. Phys. Rev. E, 86(4):040106, October 2012. 
[27] J. M. Horowitz and K. Jacobs. Quantum effects improve the energy efficiency of feedback control. Phys. Rev. E, 89(4):042134, 2014.

[28] Thomas M. Cover and Joy A. Thomas. Elements of Information Theory. Wiley-Interscience, New York, second edition, 2006.

[29] A. Dembo and O. Zeitouni. Large deviations techniques and applications, volume 38 of Stochastic Modelling and Applied Probability. Springer-Verlag, Berlin Heidelberg, 2010.

[30] Takahiro Sagawa and Masahito Ueda. Minimal Energy Cost for Thermodynamic Information Processing: Measurement and Information Erasure. Phys. Rev. Lett., 102(25):250602, June 2009.

[31] Léo Granger and Holger Kantz. Thermodynamic cost of measurements. Phys. Rev. E, 84(6):061110, December 2011.

[32] Léo Granger and Holger Kantz. Differential Landauer's principle. EPL, 101(5):50004, March 2013.

[33] Aki Kutvonen, Jonne Koski, and Tapio Ala-Nissila. Thermodynamics and efficiency of an autonomous on-chip Maxwell's demon. Scientific Reports, 6:21126, February 2016.

[34] J. V. Koski, A. Kutvonen, I. M. Khaymovich, T. Ala-Nissila, and J. P. Pekola. On-Chip Maxwell's Demon as an Information-Powered Refrigerator. Physical Review Letters, 115(26), December 2015.

[35] K. H. Kim and H. Qian. Fluctuation theorems for a molecular refrigerator. Phys. Rev. E, 75(2):022102, 2007.

[36] T. Munakata and M. L. Rosinberg. Entropy production and fluctuation theorems under feedback control: the molecular refrigerator model revisited. J. Stat. Mech., page P05010, 2012.

[37] M. Bauer, D. Abreu, and U. Seifert. Efficiency of a brownian information machine. J. Phys. A: Math. Theor., 45:162001, 2012.

[38] Jordan M. Horowitz, Takahiro Sagawa, and Juan M. R. Parrondo. Imitating Chemical Motors with Optimal Information Motors. Phys. Rev. Lett., 111(1):010602, July 2013.

[39] H. Sandberg, J.-C. Delvenne, N. J. Newton, and S. K. Mitter. Maximum work extraction and implementatio costs for nonequilibrium maxwell's demons. Phys. Rev. E, 90:042119, 2014.

[40] Jordan M. Horowitz and Henrik Sandberg. Second-law-like inequalities with information and their interpretations. New J. Phys., 16(12):125007, December 2014. 